\title{
\begin{tabular}{l|l} 
MitTraries & DSpace@MIT
\end{tabular}
}

\author{
MIT Open Access Articles
}

\section{L2 Gain Stability of Switched Output Feedback Controllers for a Class of LTI Systems}

The MIT Faculty has made this article openly available. Please share how this access benefits you. Your story matters.

Citation: Santarelli, K.R., and M.A. Dahleh. “L2 Gain Stability of Switched Output Feedback Controllers for a Class of LTI Systems." Automatic Control, IEEE Transactions on 54.7 (2009): 1504-1514. (C) 2009 IEEE

As Published: http://dx.doi.org/10.1109/tac.2009.2022096

Publisher: Institute of Electrical and Electronics Engineers

Persistent URL: http://hdl.handle.net/1721.1/54767

Version: Final published version: final published article, as it appeared in a journal, conference proceedings, or other formally published context

Terms of Use: Article is made available in accordance with the publisher's policy and may be subject to US copyright law. Please refer to the publisher's site for terms of use. 


\title{
L2 Gain Stability of Switched Output Feedback Controllers for a Class of LTI Systems
}

\author{
Keith R. Santarelli, Member, IEEE, and Munther A. Dahleh, Fellow, IEEE
}

\begin{abstract}
Our previous work has been devoted to designing asymptotically stabilizing switching controllers for a class of second order LTI plants. Here, we extend the results of our previous work by proving that, when a plant can be asymptotically stabilized using a particular switching architecture, the overall closed-loop interconnection is also finite $\mathrm{L2}$ gain stable. We shall first prove this result for a simplified problem in which a portion of the switching architecture has full access to the state of the plant and shall then extend to the case where the architecture only has access to the plant output by designing an appropriate observer.
\end{abstract}

Index Terms-Controller design, hybrid systems, L2 gain, output feedback, switching systems.

\section{INTRODUCTION}

$\mathbf{T}$ HE problem of stabilizing a continuous-time system via hybrid output feedback is one which has received a great amount of attention in the past decade (see, e.g., [1]-[3], [8], [11]-[13], [16], [17], [18], [19], [20]). In this paper, we are concerned with the operation of a particular switching architecture, namely the architecture depicted in Fig. 1. Here, $P(s)$ is a second order SISO LTI plant to be controlled. At every time $t$, the control input to the plant $u(t)$ is equal to either $v_{0} y(t)$ or $-v_{0} y(t)$ where $v_{0}>0$. It is the job of the block labeled "Supervisor" to produce a switching signal $\sigma(t)$ that orchestrates switching between $v_{0}$ and $-v_{0}$ in a stabilizing manner. The structure of the supervisor is indicated in part (b) of the figure. A first order observer is used to produce an estimate of the plant state (represented by $\hat{x}_{1}$ and $\hat{x}_{2}$ ). This state estimate is then input to a memoryless switching law which outputs the switching signal $\sigma(t)$.

The motivation for investigating the switching architecture of Fig. 1 stems from a general interest in the design of switching systems in which the analog control input to the plant is constrained to be simple in some sense. Such problems arise, for instance, in the electronics industry where the design of feedback compensators for operational amplifier circuits are typically no higher than first order feedback compensators ([6], [9]). We consider the problem of switching between proportional gains (the blocks labeled $v_{0}$ and $-v_{0}$ in Fig. 1) since doing

Manuscript received June 07, 2007; revised January 15, 2008. First published June 23, 2009; current version published July 09, 2009. Recommended by Associate Editor I. V. Kolmanovsky.

K. R. Santarelli is with the Electrical and Microsystems Modeling Department, Sandia National Laboratories, Albuquerque, NM 87185 USA (e-mail: krsanta@sandia.gov).

M. A. Dahleh is with the Laboratory for Information and Decision Systems, Massachusetts Institute of Technology, Cambridge, MA 02139-4307 USA (e-mail: dahleh@mit.edu).

Digital Object Identifier 10.1109/TAC.2009.2022096

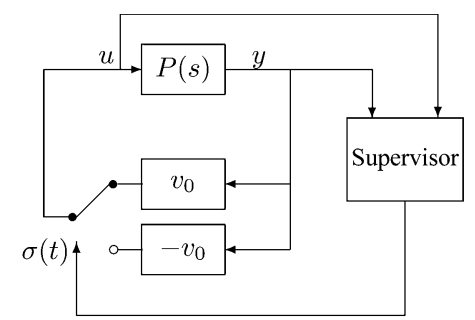

(a) Switching architecture under investigation in this paper.

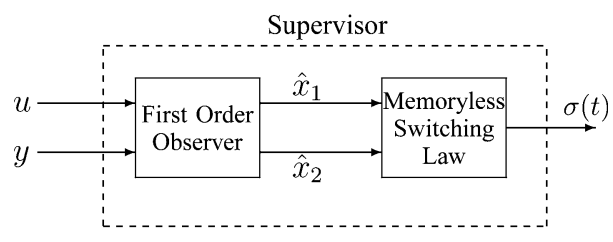

(b) Structure of supervisor in Fig. 1(a).

Fig. 1. Block diagram of switching architecture (exogenous inputs not shown).

so is simple from an implementation-level perspective, and because the closed-loop dynamics of the overall feedback interconnection are often simple enough to analyze via elementary techniques. This allows us to make concrete mathematical statements while gaining some insight into the problem-at-large of switching under constrained control actions.

It is not obvious that the switching architecture of Fig. 1 will provide any performance benefits over a first order linear controller due to the simplicity of the proportional gain analog control action, but one can show that this is indeed the case. In a separate manuscript see [25], where we provide a formal comparison between the performance of the switching architecture of Fig. 1 to two other forms of LTI control for a simple tracking problem to illustrate the potential benefits of using this switching architecture over other, more traditional forms of LTI control.

\section{A. Input-Output Stability: Problem Motivation}

Our former work has focused on the design of asymptotically stabilizing controllers; [16] provides a set of necessary and sufficient conditions for which a given second order plant is stabilizable via switched proportional gain feedback, and a specific switching control law is provided when stability is possible; [17] extends the first result by considering an optimal control problem in which the objective is to stabilize a second order LTI system via switched proportional gain feedback in a manner which maximizes the rate of convergence of the state trajectory to the origin. While such results are a necessary first step in the development of tools for analyzing and designing the switching controllers depicted in Fig. 1, the current set of results are restrictive since they apply only to second order LTI systems. In 
general, one would like to be able to design switching architectures for a broader class of systems. For instance, a natural extension of our prior work is to attempt to derive analysis and design tools for LTI systems of arbitrary dimension. Some preliminary results in this direction do exist, but we shall not focus on them here; we shall discuss this briefly in the conclusion.

Another way in which one can attempt to extend the current set of analysis and design tools to a larger set of plants is to consider the design of switching controllers of Fig. 1 for the class of plants which can be "well-approximated" by a second order LTI plant. By making use of the Small Gain Theorem, if a given plant $P$ (which, in general can be nonlinear, timevarying, and of order greater than two) is sufficiently close to a second order LTI model $\hat{P}$ where "closeness" is measured in terms of the difference $\|P-\hat{P}\|$ in some induced system norm, then one can use existing tools to design a controller for the LTI approximation $\hat{P}$ and be guaranteed that the resulting controller will stabilize the original plant $P$, as well.

As it turns out, the systems that we have studied in our previous work [16], [17] exhibit a form of input-output stability in the form of L2 gain stability. By proving that the systems of Fig. 1 are finite $\mathrm{L} 2$ gain stable for classes of plants $P(s)$ that are second order LTI systems, we will effectively prove that any plant that is sufficiently well-modelled by an appropriate second order LTI system $\hat{P}(s)$ in an L2 gain sense can be stabilized via the techniques we have already developed.

The main goal of this paper is to prove that, under certain mild assumptions on the parameters of the plant and switching gain $v_{0}$, the algorithms developed in [17] for asymptotic stability are also finite L2 gain stabilizing when exogenous inputs are added to the system dynamics. While we also have results which show how to extend our existing design techniques to systems which have a good second order LTI approximation in an L2 gain sense, we refer the reader to [18] for the details of this extensions (including a specific higher order design example) due to space constraints.

\section{B. Related Work}

While there exists a large amount of work in the literature related to the study of switching systems, to the knowledge of the authors, the number of results related to the specific question of L2 gain bounds for switched system are few. The switching laws we presented in [16], off of which we base the switching laws we shall examine in this document, are in the same general family of switching laws as those presented by Xu et al. in [19], [20]. In this work, the authors consider a set of state-dependent switching laws that switch between a given set of vector fields. The work presented by $\mathrm{Hu}$ et al. in [8] extends the work of [19], [20] to a case more similar to the one we consider here: the vector fields are not fixed but are, rather, designed by implementing a switched output feedback law that switches between two scalar gains. While the results in these papers are constructive, to the knowledge of the authors, the domain of these results has not been extended outside of the realm of asymptotic stability. A similar statement can be made regarding work on designing hybrid output feedback automata for second order linear systems (see, e.g., the work of Benassi et al. in [2] or Litsyn et al. in [12])
Nevertheless, certain classes of switched systems have been studied in recent years which provide positive results on L2 gain stability. Both Hespanha in [7] and Zhai et al. in [21], [22] consider the problem of establishing finite L2 gains for systems that switch between Hurwitz/Schur matrices. In [5], Gonçalves considers an optimal design problem to minimize the L2 gain of a class of on-off systems in which the presence of the exogenous input does not affect the associated switching times. By contrast, the work we present here requires neither of these assumptions; the exogenous inputs are allowed to affect the sequence of switching times between subsystems, and the subsystems between which switching takes place are not required to be individually stable (in fact, while we will not discuss this here, it is often-times the case that instability of at least one of the subsystems is desirable for good performance-see, e.g., [18, Ch.5] and the design example presented therein)

Some recent work on L2 gain of switched systems for more general classes of nonlinear systems has been studied by Zhao et al. in [23]. In this paper, the authors formulate a set of necessary and sufficient conditions for the existence of a stabilizing switching law (for a given set of vector fields) in the form of certain Lyapunov-like functions that satisfy a set of conditions. It should be noted that these results are not constructive since they provide no guaranteed way of finding such Lyapunov-like functions when they exist (though the paper does provide some suggestions on candidate function choices, these suggestions are not guaranteed to work). By contrast, in the work we examine here, we shall prove that the class of systems we consider is guaranteed to be finite L2 gain stable by providing an explicit class of storage functions which can be used to prove finiteness of L2 gain. Moreover, we can find numerical upper bounds on the L2 gain using standard techniques of semidefinite programming [24].

We shall now briefly review some basic concepts related to L2 gain, including its definition and the concept of storage functions. We shall conclude this section by providing an outline of the remainder of the paper.

\section{L2 Gain: Definition and Generalized Storage Functions}

Recall that the L2 gain of a system $S$ is given by the following definition [14]:

Definition: The $L 2$ gain of a continuous-time system $S$ with input $w$ and output $y$ is the smallest (infimal) value of $\gamma \geq 0$ such that

$$
\inf _{T \geq 0} \int_{0}^{T}\left(\gamma^{2}\|w(t)\|^{2}-\|y(t)\|^{2}\right) d t>-\infty
$$

for all input/output pairs $y=S(w)$ where $w$ and $y$ are square integrable over arbitrary finite intervals.

A system with input $w$ and output $y$ is, therefore, said to be finite L2 gain stable if it has finite L2 gain $\gamma \geq 0$. A convenient method of proving that a system has finite L2 gain is to prove existence of a (generalized) storage function [15]. Specifically, a storage function $V(x)$ with quadratic supply rate $\sigma(w, x)$ is a positive definite function which satisfies the relationship

$$
\int_{0}^{t} \sigma(w(t), x(t)) d t \geq V(x(t))-V(x(0))
$$


where $x$ is the system state, and $w$ is an exogenous input. If we consider the quadratic supply rate $\sigma(w, x)=\gamma^{2}\|w\|^{2}-\|y\|^{2}$ where $y=h(x, w)$ is a system output, then it is well-known that existence of a generalized storage function $V(\cdot)$ is a necessary and sufficient condition for finite L2 gain stability (see, e.g., [15]).

\section{Document Outline}

A basic outline of this paper is as follows. We shall first briefly revisit some of our prior work on the design of asymptotically stabilizing controllers using the switching architecture of Fig. 1 to provide some necessary background for the remainder of the paper. Our main task will to be prove finite L2 gain stability of the systems in Fig. 1. The proof of this result will be broken down into three main steps:

1) Proving existence of a Lyapunov function (when all exogenous inputs are zero) for a simplified version of the system in Fig. 1 in which the memoryless switching law has access to the true state of the plant.

2) Using the Lyapunov function of item 1 to prove existence of a generalized storage function for finite L2 gain stability, again for the simplified case that the memoryless switching law has access to the true state of the plant.

3) Designing an appropriate first order observer and proving that the system of Fig. 1 is finite L2 gain stable.

Due to space constraints, several proofs are curtailed or omitted. When necessary, reference will be made to a more detailed version of this paper ([18, Ch.4]).

\section{BACKGROUND: ASYMPTOTICALLY STABILIZING CONTROL LAWS}

Our previous work [16], [17] and Chapter 3 of [18] deals with a simplified variant of the system depicted in Fig. 1 in which the memoryless switching law has access to the true state of the plant $x$ rather than an estimate of the plant state $\hat{x}$. While we shall not describe this work in full detail, we shall highlight the major results that allow us to design asymptotically stabilizing memoryless switching laws in what follows. The basic problem that is considered in Chapter 3 of [18] is the following: consider a second order LTI plant of the form

$$
\begin{aligned}
& \dot{x}=A x+B u \\
& y=C x
\end{aligned}
$$

where we assume that the plant is of relative degree two. We wish to design a memoryless switching law $v(x)$ which satisfies the following conditions:

1) The range of $v(x)$ is bounded, i.e., $v(x) \in\left[-v_{0}, v_{0}\right]$ for some $v_{0}>0$.

2) The control law $u=v(x) y$ yields an asymptotically stable closed-loop system in the sense that $\dot{x}=(A+v(x) B C) x$ is asymptotically stable.

3) The corresponding rate of convergence $R\left(v_{0}\right)$ given by

$$
R\left(v_{0}\right)=\min _{\|x(0)\|=1} \liminf _{T \rightarrow \infty}-\frac{1}{2 T} \ln \left(\|x(T)\|^{2}\right)
$$

is as large as possible.

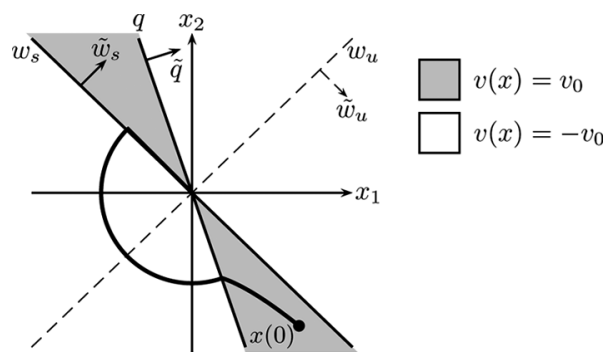

Fig. 2. Graphical illustration of the switching law of (3). The vector $w_{u}$ represents the eigenvector corresponding to the maximum eigenvalue of the matrix $\left(A+v_{0} B C\right)$, and $\tilde{w}_{u}$ is a normal vector to this eigenvector $\left(\tilde{w}_{u}^{\prime} w_{u}=0\right.$.).

The real parameter $v_{0}$ in item 1 above plays the role of the symmetric switching gain shown in Fig. 1. If $v_{0}$ is chosen sufficiently large (determined by a function of the parameters of the $A, B$, and $C$ matrices), then the following are true:

1) The maximum rate of convergence, denoted by $R^{*}\left(v_{0}\right)$, is given by

$$
R^{*}\left(v_{0}\right)=-\lambda_{\min }\left(A+v_{0} B C\right)>0
$$

where $\lambda_{\min }(\cdot)$ denotes the smallest eigenvalue of a square matrix.

2) Let $w_{s}$ represent the eigenvector corresponding to the minimum eigenvalue of the matrix $A+v_{0} B C$, and let $\tilde{w}_{s}$ and be a vector such that $\tilde{w}_{s}^{\prime} w_{s}=0$. Then there exists a vector $\tilde{q}$ such that the memoryless switching law

$$
v(x)=\left\{\begin{array}{cc}
v_{0} & x^{\prime}\left(\tilde{w}_{s} \tilde{q}^{\prime}\right) x \leq 0 \\
-v_{0} & x^{\prime}\left(\tilde{w}_{s} \tilde{q}^{\prime}\right) x>0
\end{array}\right\}
$$

makes the dynamical system $\dot{x}=(A+v(x) B C) x$ globally exponentially stable with rate $R^{*}\left(v_{0}\right)$.

A graphical illustration of the control law $v(x)$ in item 2 is depicted in Fig. 2. Note that if the initial condition $x(0)$ lies along $w_{s}$, then, by choosing a control law with $v\left(w_{s}\right)=v_{0}$, the state trajectory will evolve as

$$
x(t)=e^{\lambda_{\min }\left(A+v_{0} B C\right) t} x(0)
$$

which converges to the origin exponentially with rate $-\lambda_{\min }\left(A+v_{0} B C\right)$. The basic principle behind the design of the overall control law $v(x)$ is, hence, the following: if the initial condition $x(0)$ does not initially lie along the manifold spanned by $w_{s}$, design $v(x)$ such that the state trajectory will reach this manifold in finite time. By choosing any vector $q$ that lies "between" $w_{s}$ and $w_{u}$ (which is the eigenvector corresponding to the maximum eigenvalue of $A+v_{0} B C$ ), we can find a vector $\tilde{q}$ that is normal to $q$ such that the control law of (3) achieves this goal. A sample phase portrait is illustrated in Fig. 2. Here, the initial condition $x(0)$ lies in the region where $v(x)=v_{0}$. Thus, the system dynamics initially evolve according to $\dot{x}=\left(A+v_{0} B C\right) x$. In finite time, the state trajectory is driven onto the manifold spanned by the vector $q$ at which time $v(x)$ switches from $v_{0}$ to $-v_{0}$. The eigenvalues of the matrix $A-v_{0} B C$ are designed to be complex-valued, hence, rotation is induced, and the the state trajectory is driven onto the manifold spanned by $w_{s}$ in finite time. 
The vector $q$ (and, correspondingly, $\tilde{q}$ ) is a free parameter; hence, the control law of (3) is not unique. The interested reader is referred to Chapter 3 of [18] for a discussion of ways of selecting the vectors $q$ and $\tilde{q}$.

For the next several sections, we shall assume a particular state-space representation for the plant and will prove finite L2 gain stability within the framework of this particular set of coordinates. We shall then provide a proof for arbitrary state-space descriptions via a change of coordinates.

As mentioned above, we consider second order LTI plants of relative degree two, i.e., plants of the form

$$
P(s)=\frac{c}{s^{2}+a s+b}
$$

with $a, b, \in \mathbf{R}$. Note that, without loss of generality, we may take $c=1$. In this case, we may describe the dynamics of the plant via

$$
\begin{aligned}
{\left[\begin{array}{c}
\dot{x}_{1} \\
\dot{x}_{2}
\end{array}\right] } & =\left[\begin{array}{cc}
-\frac{a}{2} & \mu \\
\frac{-b+\frac{a^{2}}{4}}{\mu} & -\frac{a}{2}
\end{array}\right]\left[\begin{array}{l}
x_{1} \\
x_{2}
\end{array}\right]+\left[\begin{array}{c}
0 \\
\frac{1}{\mu}
\end{array}\right] u \\
y & =x_{1}
\end{aligned}
$$

where $\mu=\sqrt{a^{2} / 4-b+v_{0}}$, and where $v_{0}$ satisfies the condition $^{1}$

$$
v_{0}>\max \left\{\left|\frac{a^{2}}{4}-b\right|, 2 b-\frac{a^{2}}{2}, b\right\} .
$$

For convenience, we shall abbreviate the closed-loop dynamics of the system $\dot{x}=(A+v(x) B C) x$ in the following way:

$$
\dot{x}= \begin{cases}A_{1} x & x^{\prime}\left(\tilde{w}_{s} \tilde{q}^{\prime}\right) x \leq 0 \\ A_{2} x & x^{\prime}\left(\tilde{w}_{s} \tilde{q}^{\prime}\right) x>0\end{cases}
$$

where the matrices $A_{1}$ and $A_{2}$ are given by

$$
A_{1}=\left[\begin{array}{cc}
-\frac{a}{2} & \mu \\
\mu & -\frac{a}{2}
\end{array}\right], \quad A_{2}=\left[\begin{array}{cc}
-\frac{a}{2} & \mu \\
\mu-\frac{2 v_{0}}{\mu} & -\frac{a}{2}
\end{array}\right] .
$$

\section{CONSTRUCTION OF LyAPUNOV FUNCTION}

In Chapter 3 of [18], we proved that the closed-loop system described by (7) is exponentially stable when $q$ (and, correspondingly $\tilde{q}$ ) is appropriately chosen and, hence, admits a Lyapunov function $V(x)$ which is monotonically decreasing along the system trajectories. Assuming, for the moment, that a smooth Lyapunov function exists, then it will satisfy the conditions

$$
\begin{array}{ll}
-\nabla V(x) A_{1} x>0 & x^{\prime}\left(\tilde{w}_{s} \tilde{q}^{\prime}\right) x \leq 0 \\
-\nabla V(x) A_{2} x>0 & x^{\prime}\left(\tilde{w}_{s} \tilde{q}^{\prime}\right) x>0 .
\end{array}
$$

While the above conditions are sufficient for proving asymptotic stability, by themselves, they are not sufficient for finding a storage function when exogenous inputs are added to the system. To illustrate one of the key issues that arises by trying to use a Lyapunov function that only satisfies the conditions of

\footnotetext{
${ }^{1}$ See Chapter 3 of [18] for the origins of this condition.
}

(9) and (10) as a model for a storage function, consider the case when the switching law $v(x)$ evolves according to

$$
v(x)= \begin{cases}v_{0} & (x+g)^{\prime}\left(\tilde{w}_{s} \tilde{q}^{\prime}\right)(x+g) \leq 0 \\ -v_{0} & (x+g)^{\prime}\left(\tilde{w}_{s} \tilde{q}^{\prime}\right)(x+g)>0\end{cases}
$$

where $g=g(t)$ is an exogenous input. It is clear that, for any value of $x$ in the state-space, a value of $g$ can always be chosen so as to "fool" the supervisor, e.g., $g$ is such that $(x+$ $g)^{\prime}\left(\tilde{w}_{s} \tilde{q}^{\prime}\right)(x+g) \leq 0$ but $x^{\prime}\left(\tilde{w}_{s} \tilde{q}^{\prime}\right) x>0$. Without corruption, the dynamics would evolve according to $\dot{x}=A_{2} x$, but, instead, they evolve according to $\dot{x}=A_{1} x$, and it is quite possible that $-\nabla V(x) A_{1} x<0$. When $g$ is small compared to $x$ (so that $x$ lies close in angle to either $q$ or $w_{s}$ ), this is particularly problematic since, informally speaking, positivity of the expression

$$
\gamma^{2}\|g\|^{2}-\|y\|^{2}-\frac{d}{d t} V(x(t))
$$

essentially reduces to positivity of $-\nabla V(x) A_{1} x$ for small $g$.

We attempt to fix the above problem in the following way: in addition to requiring that $-\nabla V(x) A_{1} x>0$ whenever $x^{\prime}\left(\tilde{w}_{s} \tilde{q}^{\prime}\right) x \leq 0$ and $-\nabla V(x) A_{2} x>0$ whenever $x^{\prime}\left(\tilde{w}_{s} \tilde{q}^{\prime}\right) x<0$, we wish to find a Lyapunov function for which $-\nabla V(x) A_{i} x>0$ for both $i=1,2$ whenever $x$ is close to either $q$ or $w_{s}$ in an angular sense. While it is not obvious, if we prove existence of a Lyapunov function which satisfies these additional requirements, we shall be able to augment it into a storage function to prove that the systems under study have finite L2 gain.

The main goal of this section is to prove that there exists a piecewise differentiable Lyapunov function for the system of (7) which satisfies the above additional properties. In our effort to prove this, we shall actually find a Lyapunov function for a different (but related) auxiliary system and shall then prove that the Lyapunov function we found for the different system also acts as a Lyapunov function for the first system with the specified additional requirements.

\section{A. Auxiliary Switching System}

Before we begin our discussion of the new switching system we wish to study, we shall re-describe (7) in polar coordinates as it provides a more convenient description for this section. The radial component $r$ and the angular component $\theta$ can be described via the following dynamics:

$$
\left[\begin{array}{l}
\dot{r} \\
\dot{\theta}
\end{array}\right]= \begin{cases}f_{1}(r, \theta) & \phi_{q} \leq \theta \leq \frac{3 \pi}{4} \text { or } \\
& \phi_{q}+\pi \leq \theta \leq \frac{7 \pi}{4} \\
f_{2}(r, \theta) & \text { otherwise }\end{cases}
$$

where $\phi_{q} \in(\pi / 4,3 \pi / 4)$ is the angle that the vector $q$ makes with respect to the positive $x_{1}$ axis, and the functions $f_{1}(r, \theta)$ and $f_{2}(r, \theta)$ are given by

$$
\begin{aligned}
& f_{1}(r, \theta)=\left[\begin{array}{c}
-\frac{a}{2} r+\mu r \sin 2 \theta \\
\mu \cos 2 \theta
\end{array}\right] \\
& f_{2}(r, \theta)=\left[\begin{array}{c}
-\frac{a}{2} r+\left(\mu-\frac{v_{0}}{\mu}\right) r \sin 2 \theta \\
\left(\mu-\frac{v_{0}}{\mu}\right) \cos 2 \theta-\frac{v_{0}}{\mu} .
\end{array}\right] .
\end{aligned}
$$




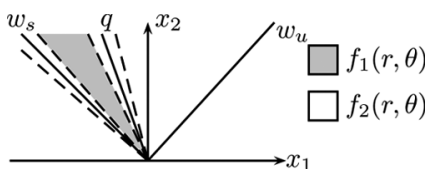

(a) $\phi_{q}>\pi / 2$

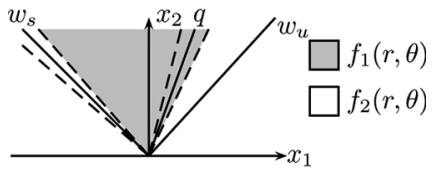

(b) $\phi_{q}<\pi / 2$

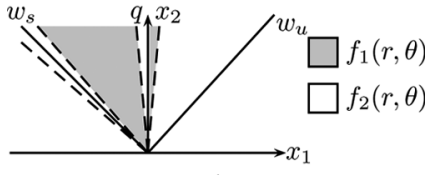

(c) $\phi_{q}=\pi / 2$

Fig. 3. Auxiliary switching system for the different values of $\phi_{q}$. The dashed lines each make an angle of $\phi_{0}$ with $w_{s}$ and $q$, and the system dynamics between the pairs of dashed lines surrounding $w_{s}$ and $q$, respectively, are chosen to maximize $\dot{r}$.

For a given value of $\theta$, consider now the problem of determining the vector field $f_{1}$ or $f_{2}$ which maximizes $\dot{r}$. Since $\mu>$ $\mu-v_{0} / \mu$, we see that $f_{1}$ maximizes $\dot{r}$ for $\theta$ lying in the first and third quadrants, while $f_{2}$ maximizes $\dot{r}$ for $\theta$ lying in the second and fourth quadrants. The new switching system that we shall study has dynamics which can be described as follows. The values of $\theta$ for which we choose to use $f_{1}$ and $f_{2}$ in (11) will be almost exactly the same in our new system, with the following exception: around the switchings boundaries $w_{s}$ (corresponding to $\theta$ values of $3 \pi / 4$ and $7 \pi / 4$ ) and $q$ (corresponding to $\theta$ values of $\phi_{q}$ and $\phi_{q}+\pi$ ), we shall construct a small cone for which the system dynamics will evolve according to whichever vector field maximizes $\dot{r}$. Depending upon the value of $\phi_{q}$, this gives rise to three possible situations which are depicted graphically in Fig. 3. When $\phi_{q}>\pi / 2$, we consider a system which evolves according to the dynamics

$$
\left[\begin{array}{l}
\dot{r} \\
\dot{\theta}
\end{array}\right]= \begin{cases}f_{1}(r, \theta) & \phi_{q}+\phi_{0} \leq \theta \leq \frac{3 \pi}{4}-\phi_{0} \text { or } \\
& \phi_{q}+\phi_{0}+\pi \leq \theta \leq \frac{7 \pi}{4}-\phi_{0} \\
f_{2}(r, \theta) & \text { otherwise }\end{cases}
$$

where $\phi_{0}>0$ is a small angle. We arrived at this description in the following way: we first investigate the cone described by $3 \pi / 4-\phi_{0} \leq \theta \leq 3 \pi / 4+\phi_{0}$ (depicted in part (a) of Fig. 3 as the sector formed by the two dotted lines that surround $w_{s}$ ) and consider the task of choosing the vector field that maximizes $\dot{r}$ in this region. From the analysis in the previous paragraph, we see that $f_{2}$ maximizes $\dot{r}$ everywhere in a small cone, so we choose this vector field in the small cone. Similarly, we construct a small cone about $q\left(\phi_{q}-\phi_{0} \leq \theta \leq \phi_{q}+\phi_{0}\right)$ and, again, choose the vector field which maximizes $\dot{r}$ in this region. For $\phi_{q}>$ $\pi / 2$, we find that, again, $f_{2}$ maximizes $\dot{r}$ everywhere in this cone. When $\phi_{q}$ is less than $\pi / 2$, it turns out that $f_{1}$ maximizes $\dot{r}$ everywhere in the cone (depicted in part (b) of the figure), and the corresponding switching system dynamics are described via

$$
\left[\begin{array}{c}
\dot{r} \\
\dot{\theta}
\end{array}\right]= \begin{cases}f_{1}(r, \theta) & \phi_{q}-\phi_{0} \leq \theta \leq \frac{3 \pi}{4}-\phi_{0} \text { or } \\
& \phi_{q}-\phi_{0}+\pi \leq \theta \leq \frac{7 \pi}{4}-\phi_{0} \\
f_{2}(r, \theta) & \text { otherwise. }\end{cases}
$$

A similar description can be written for the case when $\phi_{q}=\pi / 2$ and is depicted graphically in part (c) of Fig. 3 .

As we shall discuss shortly, for $\phi_{0}$ sufficiently small, the switching systems described above are exponentially stable in all three cases. We shall formally prove results only for the case when $\phi_{q}>\pi / 2$ as the proofs for the other two cases are similar.

It is clear that if the auxiliary switching system is stable, it admits a Lyapunov function that proves stability. It is also clear that if the auxiliary switched system is stable, then the original system is stable as well. What is not necessarily clear-and which we shall spend a fair bit of time proving at the end of this section-is that if one finds a Lyapunov function of a particular form for the auxiliary system, this Lyapunov function is also a Lyapunov function for the original system which satisfies the additional criterion that the Lyapunov function is decreasing for both vector fields in a small cone around $w_{s}$ and in a small cone around $q$.

\section{B. Exponential Stability of the Auxiliary Switching System}

One can prove exponential stability of the auxilliary switching system of (12) via the method of Poincaré maps [10]. We omit a formal proof here due to its length (one can be found in Chapter 4 of [18]), but we shall outline a sketch of this proof, considering the specific case where $\phi_{q}>\pi / 2$. Examining the top portion of Fig. 3, we see there is a cone in which the dynamics evolve according to $f_{1}(r, \theta)$, one of whose boundaries lies very close to the stable eigenvector $w_{s}$. One can show formally that the closer the left-most boundary of the cone lies to the stable eigenvector, the more the phase portraits will exhibit initial shrinkage in the Euclidean norm before they leave this cone. More specifically, the initial shrinkage in the Euclidean norm that the phase portraits experience before they leave the cone governed by $f_{1}(r, \theta)$ can be made arbitrarily small as $\phi_{0}$ tends to zero. Moreover, one can show that the growth in the Euclidean norm due to the vector field $f_{2}(r, \theta)$ is bounded, and that any phase portrait must pass through any given ray periodically. Combining these facts allows us to conclude that, for all trajectories of the auxiliary switching system of (12), there exist times $t_{0}>0$ and $T>0$ and $\alpha \in(0,1)$ such that $r\left(t_{0}+k T\right) \leq \alpha^{k} r\left(t_{0}\right)$ from which we conclude that $r\left(t_{0}+k T\right) \rightarrow 0$ as $k \rightarrow \infty$. Using a continuity argument, one can then show that $r(t) \rightarrow 0$ as $t \rightarrow \infty$.

\section{Construction of Piecewise Differentiable Lyapunov Function for Auxiliary System}

We shall now prove that the auxiliary switching system has a Lyapunov function which is strictly decreasing along system trajectories and which is piecewise differentiable. We shall prove these statements only for the case when $\phi_{q}>\pi / 2$ (the other two cases can be considered similarly). The first formal statements that we shall utilize are the following:

Proposition III.1: Consider the system

$$
\dot{x}=f(x)= \begin{cases}A_{1} x & x^{\prime} F_{1} F_{2}^{\prime} x \leq 0 \\ A_{2} x & x^{\prime} F_{1} F_{2}^{\prime} x>0\end{cases}
$$

where $A_{1}$ and $A_{2}$ are as in (8), $F_{1}$ is the clockwise-oriented normal vector to the ray $\theta=3 \pi / 4-\phi_{0}$, and $F_{2}$ is the clockwise- 
oriented normal vector to the ray $\theta=\phi_{q}+\phi_{0}$. Then for $\phi_{0}$ sufficiently small, the function

$$
V\left(x_{0}\right)=\int_{0}^{\infty}\|x(\tau)\|^{2} d \tau
$$

where $x(t)$ is the solution to (14) with initial condition $x(0)=$ $x_{0}$ is a Lyapunov function which is strictly decreasing along the trajectories of (14).

Proposition III.2: Consider the Lyapunov function $V\left(x_{0}\right)$ of Prop. III.1. Within the interior of each cone $x^{\prime} F_{1} F_{2}^{\prime} x \leq 0$ and $x^{\prime} F_{1} F_{2}^{\prime} x>0$ where $F_{1}$ and $F_{2}$ are given as in Prop. III.1, the partial derivatives $\partial V / \partial x_{1}$ and $\partial V / \partial x_{2}$ exist and are continuous.

We omit the proof of these statements as they follow from common results concerning the existence of Lyapunov functions for exponentially stable systems and basic results from functional analysis, but the interested reader can find proofs of both statements in Chapter 4 of [18]. The result of these propositions yields the following theorem, whose proof can be found in the Appendix :

Theorem III.1: Consider the function $V(\cdot)$ of Prop. III.1 where $\phi_{0}$ is sufficiently small to guarantee that $V\left(x_{0}\right)$ is a Lyapunov function for the system of (12). Then $V(\cdot)$ is a Lyapunov function for the original system of (7) and satisfies the conditions

$$
\begin{array}{ll}
-\nabla V(x) A_{1} x>0, & x^{\prime} \tilde{w}_{s}^{\prime} \tilde{q} x \leq 0 \\
-\nabla V(x) A_{2} x>0, & x^{\prime} \tilde{w}_{s}^{\prime} \tilde{q} x>0 .
\end{array}
$$

Moreover, there exists a small cone about the vectors $w_{s}$ and $q$ for which the Lyapunov function is decreasing along both vector fields, i.e.,

$$
-\nabla V(x) A_{i} x>0, \quad x^{\prime} H_{1 i} H_{2 i}^{\prime} x \leq 0
$$

for $i=1,2$, where $A_{i}$ are given as in (8), $H_{11}$ and $H_{21}$ are clockwise-oriented normal vectors to the rays $\theta=3 \pi / 4-\phi_{1}$ and $\theta=3 \pi / 4+\phi_{1}$, and where $H_{21}$ and $H_{22}$ are clockwiseoriented normal vectors to the rays $\theta=\phi_{q}-\phi_{1}$ and $\theta=\phi_{q}+\phi_{1}$ where $\phi_{1}>0$ is sufficiently small.

\section{L2 GAin STABility: Full State INFORMATion}

We are now ready to consider the problem of proving that the systems under investigation are finite L2 gain stable. Specifically, we wish to investigate the following setup: consider signals $g_{i}(t)$ for $i=1,2$, and 3 and consider the following system dynamics:

$$
\begin{aligned}
{\left[\begin{array}{c}
\dot{x}_{1} \\
\dot{x}_{2}
\end{array}\right] } & =\left[\begin{array}{cc}
-\frac{a}{2} & \mu \\
\frac{-b+\frac{a^{2}}{4}}{\mu} & -\frac{a}{2}
\end{array}\right]\left[\begin{array}{l}
x_{1} \\
x_{2}
\end{array}\right]+\left[\begin{array}{l}
0 \\
\frac{1}{\mu}
\end{array}\right] u^{\prime} \\
y & =x_{1}
\end{aligned}
$$

where, as before, $\mu=\sqrt{a^{2} / 4-b+v_{0}}$, and where $u^{\prime}=u+g_{3}$. We now consider a control law of the form $u(z)=v(z) y$ where $z=x+\hat{g}$ with $\hat{g}=\left[\begin{array}{ll}g_{1}(t) & g_{2}(t)\end{array}\right]^{\prime}$, and where $v(z)$ is given by

$$
v(z)=\left\{\begin{array}{rr}
v_{0} & z^{\prime}\left(\tilde{w}_{s} \tilde{q}^{\prime}\right) z \leq 0 \\
-v_{0} & z^{\prime}\left(\tilde{w}_{s} \tilde{q}^{\prime}\right) z>0
\end{array}\right.
$$

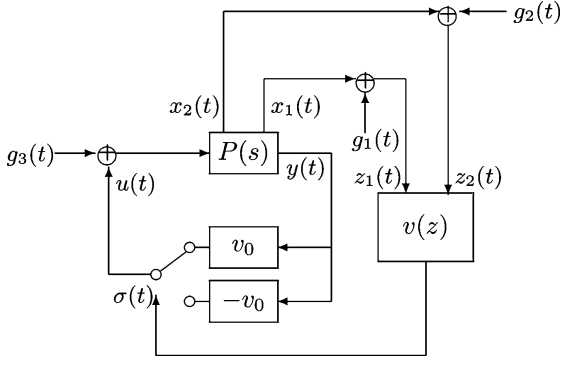

Fig. 4. Block diagram depicting where the exogenous signals $g_{1}(t), g_{2}(t)$, and $g_{3}(t)$ enter into the system dynamics for the full state L2 gain problem.

If we define $g \triangleq\left[\begin{array}{lll}g_{1}(t) & g_{2}(t) & g_{3}(t)\end{array}\right]^{\prime}$, the formal statement that we wish to prove in this section is the following: under the assumptions on $v_{0}$ given by (6), for any matrices $E \in \mathbf{R}^{m \times 2}$ and $F \in \mathbf{R}^{m \times 3}$ where $m \in \mathbf{Z}^{+}$, there exists $\gamma>0$ such that

$$
\inf _{T>0} \int_{0}^{T}\left(\gamma^{2}\|g\|^{2}-\|E x+F g\|^{2}\right) d t>-\infty .
$$

In other words, we wish to prove that the $\mathrm{L} 2$ gain from the vector $g$ to any linear combination of the state and output is finite.

Fig. 4 depicts graphically where the signals $g_{1}(t), g_{2}(t)$, and $g_{3}(t)$ enter the system dynamics. We see that $g_{1}(t)$ and $g_{2}(t)$ "corrupt" the information that is passed to the switching law $v(z)$ (which, in the absence of either $g_{1}(t)$ or $g_{2}(t)$ is equal to the optimal switching law $v(x)$ of the previous section), while $g_{3}(t)$ corrupts the control input $u(t)$. A few words on the generality of this model are in order. There are three additional places in the block diagram where exogenous inputs could be added: one at the input to the gain $v_{0}$, another at the input to the gain $-v_{0}$, and another at the output of the supervisor $v(z)$. The first two inputs can be effectively modelled by the input $g_{3}(t)$ (see [18, Ch.4] for an explanation). The last input - an input present at the output of the supervisor $v(z)$-is not a case that we shall consider, since adding a signal which takes on arbitrary values in $\mathbf{R}$ is not sensible when the output $v(z)$ is discrete-valued.

In order to prove that the system under investigation has finite L2 gain, we shall prove that there exists a storage function for the quadratic supply rate $\gamma^{2}\|g\|^{2}-\|E x+F g\|^{2}$ whenever $\gamma$ is sufficiently large. Our storage function shall be based on the Lyapunov function of the previous section (in fact, it will be a scalar multiple of it). Our proof will rely on considering two separate cases, one in which $\|g\| \leq \epsilon\|x\|$ and one in which $\|g\| \geq \epsilon\|x\|$ where $\epsilon>0$ is a sufficiently small real number. The proof for the former case will rely on the result of the following Proposition whose proof can be found in [18]:

Proposition IV.3: Consider vectors $x, g \in \mathbf{R}^{2}$ such that

$$
\operatorname{sgn}\left(x^{\prime} \tilde{w}_{s} \tilde{q}^{\prime} x\right) \neq \operatorname{sgn}\left((x+g)^{\prime} \tilde{w}_{s} \tilde{q}^{\prime}(x+g)\right)
$$

where $\operatorname{sgn}(\cdot)$ is the standard signum function. Then for every $\delta>0$, there exists $\epsilon>0$ such that if $\|g\| \leq \epsilon\|x\|$, then one of the following must hold:

$$
x^{\prime} G_{1} G_{2}^{\prime} x \leq 0 \quad \text { or } \quad x^{\prime} H_{1} H_{2}^{\prime} x \leq 0
$$

where $G_{1}$ and $G_{2}$ are clockwise-oriented normal vectors to the rays $\theta=3 \pi / 4-\delta$ and $\theta=3 \pi / 4+\delta$ and $H_{1}$ and $H_{2}$ are clockwise-oriented normal vectors to the rays $\theta=\phi_{q}-\delta$ and 
$\theta=\phi_{q}+\delta$ where $\phi_{q}$ represents the angle of the vector $q$ with respect to the positive $x_{1}$ axis.

In layman's terms, Prop. IV.3 says that if $g$ is small compared to $x$, and $x$ and $x+g$ lie in different cones, then $x$ must be close in angle to one of the boundaries, either $w_{s}$ or $q$. Equipped with Prop. IV.3, we can now prove the following important result whose proof can be found in the Appendix :

Theorem IV.2 (Finite L2 Gain Theorem): Consider the Lyapunov function $V(\cdot)$ of Prop. III.1. There exist positive constants $\alpha$ and $\gamma$ such that the function $\alpha V(x)$ is a storage function for the quadratic supply rate $\gamma^{2}\|g\|^{2}-\|E x+F g\|^{2}$ along the system trajectories described by (15), (16), and (17).

Thm. IV. 2 proves finite L2 gain for a particular coordinate description of the plant $P(s)$. However, as we shall show now, one can find storage functions to prove finite L2 gain for any minimal state-space description of the second order plant $P(s)$ of relative degree two:

1) Propostion IV.4: Consider a linear system

$$
\begin{aligned}
& \dot{x}=A x+B u+B g_{3} \\
& y=E x+F g
\end{aligned}
$$

under the control law $u(x)=v(x) y$ with

$$
v(x)= \begin{cases}v_{0} & (x+\hat{g})^{\prime} F_{1} F_{2}^{\prime}(x+\hat{g}) \leq 0 \\ -v_{0} & (x+\hat{g})^{\prime} F_{1} F_{2}^{\prime}(x+\hat{g})>0\end{cases}
$$

where $\hat{g}=\left[\begin{array}{ll}g_{1}(t) & g_{2}(t)\end{array}\right]^{\prime}$, and where $F_{j} \in \mathbf{R}^{2 \times 1}$ for $j=$ 1,2 . Suppose for some value $\gamma>0$, the quadratic supply rate $\gamma^{2}\|g\|^{2}-\|E x+F g\|^{2}$ has associated nonnegative storage function $V(x)$ where $g \triangleq\left[\begin{array}{lll}g_{1}(t) & g_{2}(t) \quad g_{3}(t)\end{array}\right]^{\prime}$. Then for any invertible $T \in \mathbf{R}^{2 \times 2}$, the system

$$
\begin{aligned}
& \dot{z}=T^{-1} A T z+T^{-1} B u+T^{-1} B g_{3} \\
& y=E T z+F g
\end{aligned}
$$

under the control law $u(z)=v(z) y$ with

$$
u(z)= \begin{cases}v_{0} & (z+\tilde{g})^{\prime} \tilde{F}_{1} \tilde{F}_{2}^{\prime}(z+\tilde{g}) \leq 0 \\ -v_{0} & (z+\tilde{g})^{\prime} \tilde{F}_{1} \tilde{F}_{2}^{\prime}(z+\tilde{g})>0\end{cases}
$$

where $\tilde{g}=T^{-1} \hat{g}$ and $\tilde{F}_{j}=T^{\prime} F_{j}$ for $j=1,2$ has quadratic supply rate $\gamma^{2}\left\|g^{\prime}\right\|^{2}-\|E T z+F g\|^{2}$ with associated nonnegative storage function $V(T z)$, where $g^{\prime}$ is given by

$$
g^{\prime}=\left[\begin{array}{cc}
T^{-1} & 0 \\
0 & 1
\end{array}\right]\left[\begin{array}{c}
\hat{g} \\
g_{3}
\end{array}\right]
$$

Proof: Immediate upon making the substitution $x=T z$.

\section{OBSERVER-BASED CONTROL}

Up until this point, we have assumed that the switching law $v$ has access to the full state of the plant in making its decision (i.e., $v=v\left(x_{1}, x_{2}\right)$ where $x_{1}$ and $x_{2}$ are the states of the plant $P(s)$ ). In practice, the supervisor may have to rely on some estimate $\left[\begin{array}{ll}\hat{x}_{1} & \hat{x}_{2}\end{array}\right]^{\prime}$ of the true state $\left[\begin{array}{ll}x_{1} & x_{2}\end{array}\right]$ to make its decisions. We shall show now that, by designing a simple observer, we can design switching controllers which do not rely on access to the full state of the plant and which yield closed-loop interconnections that are finite L 2 gain stable. We shall begin by showing that one can design a reduced-order (first order) observer to estimate the "missing" state information that is not present in the output. Once we have done this, we shall show that using the output of this observer in place of the true missing state information will still yield a finite L2 gain stable system.

\section{A. Observer Design}

The design of the observer we shall use is based upon reduced order observer theory (see Exercise 29.2 in [4]). Consider a reachable and observable second order LTI system of the form

$$
\dot{x}=A x+B u, \quad y=x_{1} .
$$

Note that the class of plants we have been studying, namely second order plants of relative degree two, always has a statespace description that can be written in the above format. Since the output is, itself, the first state of the plant, we only need to estimate the second state of the plant $\hat{x}_{2}$ in order to obtain an estimate of the full state vector.

Without loss of generality, we shall assume that the entry $A_{22}<0$. To design an observer $\hat{x}_{2}$ for $x_{2}$, we simply "repeat" the dynamics of $x_{2}$

$$
\dot{\hat{x}}_{2}=A_{21} x_{1}+A_{22} \hat{x}_{2}+B_{2} u \text {. }
$$

If we define the error signal $e(t)=x_{2}(t)-\hat{x}_{2}(t)$, we find that $e(t)$ evolves according to the dynamics $\dot{e}=A_{22} e$. Since $A_{22}<0$ by assumption, we see that the dynamics of the error are stable, i.e., $\hat{x}_{2}(t) \rightarrow x_{2}(t)$ exponentially as $t \rightarrow \infty$.

The addition of the above observer makes the order of our overall system equal to 3 where the components $x_{1}, x_{2}$, and $\hat{x}_{2}$ comprise a "natural" state vector. One can equivalently characterize the state in terms of the components $x_{1}, \hat{x}_{2}$ and $e$, instead, and the state-space description takes the form

$$
\left[\begin{array}{c}
\dot{\tilde{x}} \\
\dot{e}
\end{array}\right]=\left[\begin{array}{cc}
A & L \\
0 & A_{22}
\end{array}\right]\left[\begin{array}{l}
\tilde{x} \\
e
\end{array}\right]+\left[\begin{array}{l}
B \\
0
\end{array}\right] u
$$

where $\tilde{x}=\left[\begin{array}{ll}x_{1} & \hat{x}_{2}\end{array}\right]^{\prime}, A$ and $B$ are as in (19), and where $L$ is an appropriate matrix. When written in the above form, it is clear that $e$ is an uncontrollable mode of (21). We shall exploit this fact in the sections that follow.

\section{B. Finite L2 Gain Stability of Observer-Based Controller}

A block diagram of the closed-loop system with the observer of (20) in place is shown in Fig. 5 where $k_{1}=-A_{22}, k_{2}=A_{21}$, and $k_{3}=B_{2}$. As in the full-state problem of Fig. 4, we allow the addition of the three exogenous inputs $g_{1}(t), g_{2}(t)$, and $g_{3}(t)$, but we now also allow an additional exogenous input $w(t)$ to the input of the observer dynamics. In general, we wish to prove finite L2 gain stability when all four inputs $g_{1}, g_{2}, g_{3}$, and $w$ are present, but for now, we shall begin with the special case where $w(t)$ is identically 0 :

Theorem V.3: Consider the system depicted in Fig. 5 where, under the assumption $w(t)=0$ identically, the observer output $\hat{x}_{2}(t) \rightarrow x_{2}(t)$ exponentially as $t \rightarrow \infty$ where $x_{2}(t)$ is the second state of the second order plant $P(s)$. Assume that the "full state" system of Fig. 4 is finite L2 gain stable for the same plant $P(s)$ and switching law $v(z)$. Moreover, suppose that the 


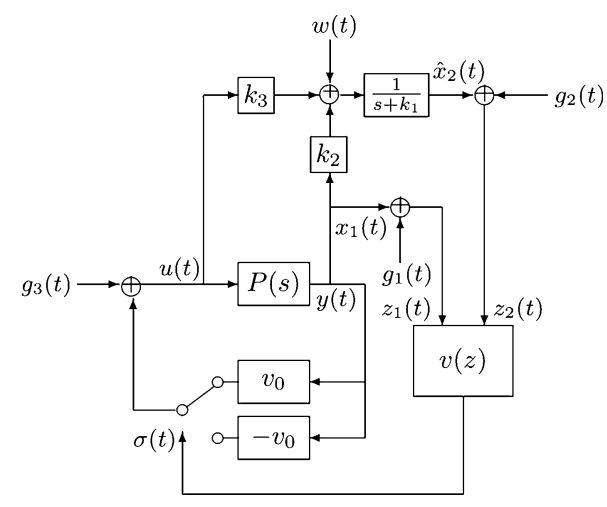

Fig. 5. Block diagram of switching system with observer in place (the observer is comprised of the blocks $1 /\left(s+k_{1}\right), k_{2}$, and $k_{3}$ with $k_{1}=-A_{22}, k_{2}=A_{21}$, and $k_{3}=B_{2}$ ). The input $w$ models the net disturbance/noise inputs to the observer.

$L_{2}$ gain from the vector $g$ to an output of the form $y=E x+F g$ in the full state system of Fig. 4 is equal to $\gamma$. Then the $L_{2}$ gain from $g$ to the output $y=E \tilde{x}+F g+M e$ in the observer-based control scheme of Fig. 5 is equal to $\gamma$ for arbitrary matrices $M$ of appropriate dimension.

The proof of this statement can be found in the Appendix .

We point out one very important corollary of the above result:

Corollary 1: When $w$ is identically 0 in Fig. 5 the $L_{2}$ gain from the vector $g$ to the output $y=E x+F g$ is the same for both the full state system of Fig. 4 and for the observer-based system of Fig. 5.

Proof (Proof of Cor. 1): With an appropriate decomposition of $E$, we may express $y$ as $E_{1} x_{1}+E_{2} x_{2}+F g$. Thm. V.3 then tells us that the L2 gain from $y$ to $E x+F g$ in the full state setup of Fig. 4 is equal to the L2 gain from $y$ to $E_{1} x_{1}+$ $E_{2} \hat{x}_{2}+M e$ for arbitrary $M$. If we select $M=E_{2}$ and notice that $\hat{x}_{2}+e=x_{2}$, we obtain the desired result.

While it is true that Cor. 1 does not hold when $w(t)$ is nonzero, we shall demonstrate that $\mathrm{L} 2$ gains for problems in which $w(t)$ is nonzero can be upper bounded by the L2 gain of a problem in which $w(t)$ is zero. We formalize this result in the following theorem:

Theorem V.4 (Observer-Based Finite L2 Gain Theorem): For the system of Fig. 5, suppose that the L2 gain from the input $g$ to an output of the form $E x+F g$ is equal to $\gamma$. Then the $\mathrm{L} 2$ gain from the composite input $\left[\begin{array}{ll}g^{\prime} & w\end{array}\right]^{\prime}$ to $E x+F g$ for the system of Fig. 6 is upper bounded by

$$
\frac{\sqrt{k_{1}^{2}+1}}{k_{1}} \gamma
$$

Proof: We begin by redrawing the block diagram of Fig. 5 in the form shown in Fig. 6. In terms of the variables in this new block diagram, define a new signal $g_{2}^{\prime}(t)=g_{4}(t)+g_{2}(t)$, and denote the L2 gain from the input vector $\tilde{g}=\left[\begin{array}{lll}g_{1} & g_{2}^{\prime} & g_{3}\end{array}\right]$ to $E x+F \tilde{g}$ as $\gamma$. We can view the overall transformation from the composite input $\left[\begin{array}{llll}g_{1} & g_{2} & g_{3} & w\end{array}\right]^{\prime}$ to the output $E x+F g$ as the cascade of two transformations, one in which we map the vector $\left[\begin{array}{ll}g^{\prime} & w\end{array}\right]^{\prime}$ into $\tilde{g}$ and one in which we map the vector $\tilde{g}$ into $E x+F \tilde{g}$. Using the submultiplicative property of L2 gains, the $\mathrm{L} 2$ gain of the overall transformation is upper bounded

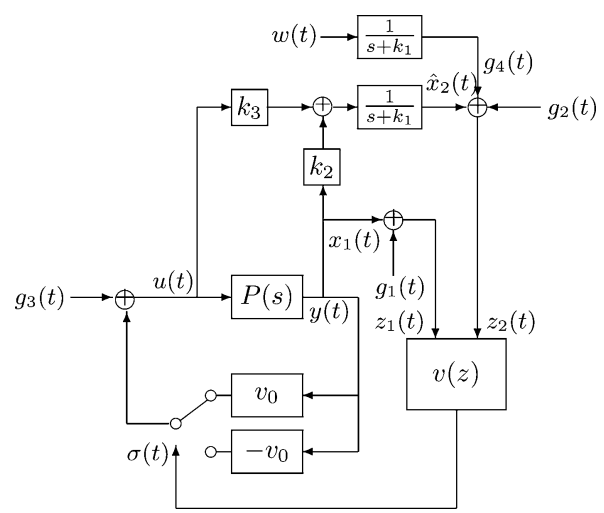

Fig. 6. Equivalent block diagram of Fig. 5 with input disturbance $w$ re-configured as an observer output disturbance $g_{4}$.

by the product of the L2 gains of these individual transformations. The map from $\left[\begin{array}{ll}g^{\prime} & w^{\prime}\end{array}\right]$ to $\tilde{g}$ is an LTI transformation $\tilde{g}=M(s)\left[\begin{array}{ll}g^{\prime} & w\end{array}\right]^{\prime}$ where

$$
M(s)=\left[\begin{array}{cccc}
1 & 0 & 0 & 0 \\
0 & 1 & 0 & \frac{1}{s+k_{1}} \\
0 & 0 & 1 & 0
\end{array}\right] .
$$

Verifying that $\|M(s)\|_{\infty}=\sqrt{k_{1}^{2}+1} / k_{1}$ completes the proof.

\section{CONCLUSION}

The main contribution of this work has been to show that, equipped with a design mechanism for an appropriate observer, the switching laws developed in our previous work [16]-[18] are finite L2 gain stable. As a consequence of this result, the design techniques of our prior work (originally just for second order LTI systems) can now be extended to all nonlinear, timevarying, and higher order plants which are well-approximated by a second order LTI system in an L2 gain sense. The interested reader is referred to [18] for more information on this (including a particular design example for a higher order system), along with details on techniques of actually computing upper bounds on the L2 gains via semidefinite programming.

Note that while we formally proved the results here for second order systems of relative degree two, this assumption is not a critical one. Indeed, preliminary work indicates that, under the assumptions made in [16], second order systems of relative degree one that are asymptotically stabilized via switching laws similar to the ones shown here are also finite L2 gain stable. Moreover, second order LTI systems with feedthrough terms can also be accommodated by augmenting the architecture of Fig. 1 with an appropriate feedforward cancellation path ([18, p.29] for details).

One of the main reasons for investigating this work is to motivate further study of switching architectures. While the results presented here are restrictive since they are derived for second order linear systems, they do open up the door for various extensions, in particular, to systems of higher dimension. Extensions to the case of switches between two linear combinations of an estimate of the plant state $x$ (i.e., implementing a control law of the form $u=K_{i} \hat{x}$, where $\hat{x}$ is the output of an observer for the true plant state $x$, and $K_{i}, i=1,2$ are appropriately selected 
gain vectors) for reachable, observable linear systems of arbitrary dimension may be tractable.

As a final note, we comment on the possibility of chattering when time delays are present. Such behavior is typically observed in sliding mode control systems. Numerical simulations indicate strongly that the control laws presented here are insensitive to chatter. This observation is a great contrast to many existing results on the design of switched static gain controllers for second order linear systems (see, e.g., [20] and figures therein) in which chatter is a fundamental characteristic of the control law rather than simply an artifact of time delays. A formal study of our switching architecture's sensitivity to chatter is an area for future research.

\section{APPENDIX}

\section{PRoOFS OF TECHNiCAL STATEMENTS}

Proof of Thm. III.1: To begin, note that in polar coordinates, $V$ of Prop. III.1 can be expressed as $V(r, \theta)=r^{2} f(\theta)$ since $V$ is homogeneous of degree 2. Moreover, the partial derivatives $\partial V / \partial r$ and $\partial V / \partial \theta$ are continuous within $x^{\prime} F_{1} F_{2}^{\prime} x \leq 0$ and $x^{\prime} F_{1} F_{2}^{\prime} x>0$ where $F_{1}, F_{2}$ are defined as in Prop. III.1.

For $\phi_{q}>\pi / 2$, by construction, the vector field of the auxiliary system and the original system are the same in the regions $x^{\prime} H_{11} H_{22}^{\prime} x \leq 0$ and $x^{\prime} H_{12} H_{21}^{\prime} x>0$ (for a graphical depiction of this, in Fig. $3, x^{\prime} H_{11} H_{22}^{\prime} x \leq 0$ corresponds to the shaded region, and $x^{\prime} H_{12} H_{21}^{\prime} x>0$ corresponds to the non-shaded region excluding the small regions containing $w_{s}$ and $q$ that are bound by the dashed lines). Hence, we have that $-\nabla V(x) A_{1} x>0$ in the region $x^{\prime} H_{11} H_{22}^{\prime} x \leq 0$ and $-\nabla V(x) A_{2} x>0$ in the region $x^{\prime} H_{12} H_{21}^{\prime} x>0$, as desired. We also have that $-\nabla V(x) A_{2} x>0$ in the regions $x^{\prime} H_{11} H_{12}^{\prime} x \leq 0$ and $x^{\prime} H_{21} H_{22}^{\prime} x \leq 0$ by virtue of the fact that $V$ is a Lyapunov function for the auxiliary system. The only thing that remains to be shown, then, is that $-\nabla V(x) A_{1} x>0$ in the regions $x^{\prime} H_{11} H_{12}^{\prime} x \leq 0$ and $x^{\prime} H_{21} H_{22}^{\prime} x \leq 0$. In polar coordinates, this amounts to showing that:

$$
\frac{\partial V}{\partial r} f_{11}(r, \theta)+\frac{\partial V}{\partial \theta} f_{12}(r, \theta)>0
$$

for $\theta \in\left[3 \pi / 4-\phi_{1}, 3 \pi / 4+\phi_{1}\right]$ and $\theta \in\left[\phi_{q}-\phi_{1}, \phi_{q}+\phi_{1}\right]$ for sufficiently small $\phi_{1}>0$, where $f_{11}(r, \theta)$ and $f_{12}(r, \theta)$ are the first and second components, respectively, of the vector field $f_{1}(r, \theta)$ of (12). Verification of the above constraint is lengthy and is omitted due to space constraints (see Chapter 4 of [18] for details).

Proof of Thm. IV.2: We wish to show that for all $x \in \mathbf{R}^{2}$ and all $g \in \mathbf{R}^{3}$ (not both identically 0 ) that there exist constants $\alpha$ and $\gamma$ both positive such that

$$
\gamma^{2}\|g\|^{2}-\|E x+F g\|^{2}-\alpha \frac{d}{d t} V(x(t))>0
$$

which can, equivalently, be expressed as

$$
\begin{aligned}
\gamma^{2}\|g\|^{2}-\|E x\|^{2}-\|F g\|^{2} & -2 x^{\prime} E^{\prime} F g \\
& -\alpha \nabla V(x)\left(A_{i} x+B g_{3}\right)>0
\end{aligned}
$$

for $i=1,2$ where $A_{i}$ are given as in (8) and where

$$
i= \begin{cases}1 & (x+\hat{g})^{\prime} \tilde{w}_{s} \tilde{q}(x+\hat{g}) \leq 0 \\ 2 & (x+\hat{g})^{\prime} \tilde{w}_{s} \tilde{q}(x+\hat{g})>0 .\end{cases}
$$

As mentioned before, we shall break the proof up into two cases: one in which $\|g\| \leq \epsilon\|x\|$ and one in which $\|g\| \geq \epsilon\|x\|$ where $\epsilon>0$ will be chosen sufficiently small.

Case 1: Small g: Consider the first case where $g$ is small compared to $x$. We wish to show first that $-\nabla V(x) A_{i} x>0$ along the system trajectories under the constraint that $\|g\| \leq$ $\epsilon\|x\|$ for $\epsilon>0$ sufficiently small. When $x^{\prime} \tilde{w}_{s} \tilde{q}^{\prime} x$ and $(x+$ $\hat{g})^{\prime} \tilde{w}_{s} \tilde{q}^{\prime}(x+\hat{g})$ have the same sign, the above statement is trivially true since $V(x)$ is a Lyapunov function for the autonomous system with no exogenous inputs. When $x^{\prime} \tilde{w}_{s} \tilde{q}^{\prime} x$ and $(x+$ $\hat{g})^{\prime} \tilde{w}_{s} \tilde{q}^{\prime}(x+\hat{g})$ are of different signs, however, this conclusion does not hold, in general, since the "wrong" vector field is being chosen. As we shall show now, however, under the assumption that $g$ is sufficiently small compared to $x$, the statement $i$ s true. First, note that by picking $\epsilon$ sufficiently small, Prop. IV.3 tells us that, whenever $x^{\prime} \tilde{w}_{s} \tilde{q}^{\prime} x$ and $(x+\hat{g})^{\prime} \tilde{w}_{s} \tilde{q}^{\prime}(x+\hat{g})$ have different signs, $x$ must lie in a small cone about either $w_{s}$ or $q$. But by Thm. III.1, there exists a small cone about $w_{s}$ and a small cone about $q$ for which $-\nabla V(x) A_{i} x>0$ for both $i=1$ and $i=2$.

Now, consider the difference

$$
-\alpha \nabla V(x) A_{i} x-\|E x\|^{2} .
$$

If we let $\eta=x /\|x\|$ for $x \neq 0$, we can express the above as

$$
\begin{aligned}
\left(-\alpha \nabla V(\eta) A_{i} \eta-\|E \eta\|^{2}\right)\|x\|^{2} & \\
& >\left(-\alpha \nabla V(\eta) A_{i} \eta-\|E\|^{2}\right)\|x\|^{2} .
\end{aligned}
$$

Now, since $\eta$ is a compact set, we have

$$
\min _{\eta, i=1,2}-\nabla V(\eta) A_{i} \eta \triangleq \beta>0
$$

and, hence

$$
\left(-\alpha \nabla V(\eta) A_{i} \eta-\|E\|^{2}\right)\|x\|^{2}>\left(\alpha \beta-\|E\|^{2}\right)\|x\|^{2}>\beta\|x\|^{2}
$$

when $\alpha>1+\|E\|^{2} / \beta$. Hence, the left-hand side of (22) can be lower bounded by

$$
g^{\prime}\left(\gamma^{2} I-F^{\prime} F\right) g-2 x^{\prime} E^{\prime} F g-\alpha \nabla V(x) B g_{3}+\beta\|x\|^{2} \text {. }
$$

For notational convenience, if we define $\tilde{\gamma}^{2}=\gamma^{2}-\lambda_{\max }\left(F^{\prime} F\right)$ for $\gamma^{2}>\lambda_{\max }\left(F^{\prime} F\right)$, we can further lower bound the above by

$$
\tilde{\gamma}^{2}\|g\|^{2}-2 x^{\prime} E^{\prime} F g-\alpha \nabla V(x) B g_{3}+\beta\|x\|^{2} \text {. }
$$

The above expression takes the form

$$
f(g, x)=\tilde{\gamma}^{2}\|g\|^{2}-2 p(x)^{\prime} g+\beta\|x\|^{2}
$$

where $p(x)$ is continuous and homogeneous of degree one. For each fixed $x \in \mathbf{R}^{2}$, the above is a quadratic form in $g$ which has minimal value

$$
-\frac{p^{2}(x)}{\tilde{\gamma}^{2}}+\beta\|x\|^{2} .
$$


If we again let $\eta=x /\|x\|$, the above can reparameterized as

$$
\left(\beta-\frac{p^{2}(\eta)}{\tilde{\gamma}^{2}}\right)\|x\|^{2}
$$

Since $\eta$ is a compact set and $p(x)$ is continuous, it follows that $\omega=\max _{\eta} p^{2}(\eta)$ exists and

$$
\left(\beta-\frac{p^{2}(\eta)}{\tilde{\gamma}^{2}}\right)\|x\|^{2}>\left(\beta-\frac{\omega}{\tilde{\gamma}^{2}}\right)\|x\|^{2}>\frac{\beta}{2}\|x\|^{2}
$$

when $\tilde{\gamma}^{2}>2 \omega / \beta$. Hence, we have shown that, under the constraint $\|g\| \leq \epsilon\|x\|$, (22) is positive for $\alpha$ and $\gamma$ sufficiently large.

Case 2: Large g: We now wish to show that if $\|g\| \geq$ $\epsilon\|x\|, \gamma$ can still be chosen sufficiently large to ensure that (22) is positive for all $x \in \mathbf{R}^{2}, g \in \mathbf{R}^{3}$ which are not both identically 0 . If we define $\tilde{\gamma}^{2}$ as in the previous section, and if we define $h(x)=\min _{i=1,2}-\alpha \nabla V(x) A_{i} x$, then the left-hand side of (22) is lower bounded by

$$
\tilde{\gamma} 2\|g\|^{2}-2 x^{\prime} E^{\prime} F g-\alpha \nabla V(x) B g_{3}-\|E x\|^{2}-h(x) .
$$

Note that the above can be written in the form

$$
\tilde{\gamma}^{2}\|g\|^{2}-2 p(x)^{\prime} g-q(x)
$$

where $p(x)$ is continuous and homogeneous of degree one and $q(x)$ is continuous and homogeneous of degree 2. Again using the parameterization $\eta=x /\|x\|$, if we consider all $g$ which satisfy $\|g\|=t\|x\|$ for some $t>0$, we can rewrite the above in the form

$$
\left(\tilde{\gamma}^{2} t^{2}-2 p(\eta)^{\prime} \tilde{g} t-q(\eta)\right)\|x\|^{2}
$$

where $\tilde{g}=g /\|g\|$. What we wish to show now that the expression in parentheses above is increasing for $t \geq \epsilon$ for all unit-length $\eta$ and $\tilde{g}$ if $\tilde{\gamma}^{2}$ is sufficiently large. Indeed, differentiating the expression in parentheses with respect to $t$ shows that the derivative is increasing whenever $t>p(\eta)^{\prime} \tilde{g} / \tilde{\gamma}^{2}$. Again, since $\eta$ and $\tilde{g}$ lie in compact sets and $p$ is continuous, $\omega=\max _{\eta, \tilde{g}} p(\eta)^{\prime} \tilde{g}$ exists, and the indicated function of $t$ is increasing whenever $t>\omega / \tilde{\gamma}^{2}$. Taking $\tilde{\gamma}^{2}>\omega / \epsilon$ guarantees that the expression in parentheses is increasing for $t \geq \epsilon$. Thus, for all $g$ which satisfy $\|g\| \geq \epsilon\|x\|$,

$$
\begin{aligned}
\left(\tilde{\gamma}^{2} t^{2}-2 p(\eta)^{\prime} \tilde{g} t-q(\eta)\right) & \|x\|^{2} \\
& >\left(\tilde{\gamma}^{2} \epsilon^{2}-2 p(\eta)^{\prime} \tilde{g} \epsilon-q(\eta)\right)\|x\|^{2} .
\end{aligned}
$$

Defining

$$
\nu=\min _{\eta, \tilde{g}}\left(-2 p(\eta)^{\prime} \tilde{g} \epsilon-q(\eta)\right)
$$

we have

$$
\left(\tilde{\gamma}^{2} \epsilon^{2}-2 p(\eta)^{\prime} \tilde{g} \epsilon-q(\eta)\right)\|x\|^{2}>\left(\tilde{\gamma}^{2} \epsilon^{2}-\nu\right)\|x\|^{2}>0
$$

for $\tilde{\gamma}^{2}>\nu / \epsilon^{2}$.

We have now shown that $\tilde{\gamma}$ (and, equivalently $\gamma$ ), can be chosen sufficiently large to ensure that (22) is positive for all $g$ and $x$ that are not both identically 0 . Hence $\alpha V(x)$ is a storage function for $\alpha$ sufficiently large, and the system has finite L2 gain.

Proof of Thm. V.3: The formal statement we wish to show is the following: suppose that for a given value of $\gamma>0$, there exists a storage function $V(x)$ for which

$$
\gamma^{2}\|g\|^{2}-\|E x+F g\|^{2}-\frac{d}{d t} V(x(t))>0
$$

along the trajectories of the system of Fig. 4. Then there exists a function $\tilde{V}(\tilde{x}, e)$ such that

$$
\gamma^{2}\|g\|^{2}-\|E \tilde{x}+F g+M e\|^{2}-\frac{d}{d t} \tilde{V}(\tilde{x}(t), e(t))>0
$$

along the trajectories of the system of Fig. 5 for arbitrary $M$ of appropriate dimension. We shall show here that the function $\tilde{V}(\tilde{x}, e)=V(\tilde{x})+p e^{2}$ is a storage function which satisfies (24) when $p>0$ is chosen sufficiently large. If we let $q=$ $-2 p A_{22}>0$, we can rewrite the lefthand side of (24) as

$$
\begin{aligned}
\gamma^{2}\|g\|^{2}- & \|E \tilde{x}+F g\|^{2}-2(E x+F g)^{\prime} M e \\
& -\|M e\|^{2}-\nabla V(\tilde{x})\left(A_{i} \tilde{x}+B g_{3}+L e\right)+q e^{2}
\end{aligned}
$$

where $A_{i}$ for $i=1,2$ are defined as in the proof of Thm. IV.2. We shall lower bound (25) by making the following observation: if the lefthand side of (23) is positive for all nonzero $g$ and $x$, then it is actually lower bounded by a function of the form $\delta\left(\|g\|^{2}+\|x\|^{2}\right)$ where $\delta>0$. To see this note, define $\delta$ to be the minimum of the lefthand side of (23) over the set $\|g\|^{2}+\|x\|^{2}=1$. The result now follows by noting that the lefthand side side of (23) is homogeneous of degree 2. Using this result, we can now conclude that (25) is lower bounded by

$$
\begin{aligned}
& \left(q-\|M\|^{2}\right) e^{2}-\left(\nabla V(\tilde{x}) L+2 \tilde{x}^{\prime} E^{\prime} M\right) e \\
& -2 g^{\prime} F M e+\delta\|\tilde{x}\|^{2}+\delta\|g\|^{2} .
\end{aligned}
$$

We shall rewrite the above in the following manner:

$$
\begin{aligned}
\frac{1}{2}\left(q-\|M\|^{2}\right) e^{2}-\left(\nabla V(\tilde{x}) L+2 \tilde{x}^{\prime} E^{\prime} M\right) e+\delta\|\tilde{x}\|^{2} \\
+\frac{1}{2}\left(q-\|M\|^{2}\right) e^{2}-2 g^{\prime} F M e+\delta\|g\|^{2} .
\end{aligned}
$$

We shall show that, for a sufficiently large choice of $q>0$, both (27) and (27) can be made positive. To prove this for (27), note that because $\nabla V(\tilde{x})$ is homogeneous of degree one, (27) can be lower bounded by

$$
\frac{1}{2}\left(q-\|M\|^{2}\right) e^{2}-\beta\|\tilde{x}\||| e \mid+\delta\|\tilde{x}\|^{2}
$$

for some $\beta>0$. Eqn. (28) is a quadratic form in the scalar quantities $\|\tilde{x}\|$ and $|e|$ and is positive for all nonzero $\tilde{x}$ and $e$ if and only if the matrix

$$
\left[\begin{array}{cc}
\delta & -\frac{\beta}{2} \\
-\frac{\beta}{2} & \frac{1}{2}\left(q-\|M\|^{2}\right)
\end{array}\right]
$$

is positive definite. A simple calculation shows that the above matrix will be positive definite if $\delta\left(q-\|M\|^{2}\right)>\beta^{2} / 2$ which can always be satisfied by choosing $q$ (and hence $p$ of the storage function $\tilde{V}(\tilde{x}, e)$ ) sufficiently large. A similar argument can be made to show that (27) can be made nonnegative for all nonzero 
$e$ and $g$ for sufficiently large $q$, as well, to show that (24) can be made positive for all nonzero $e, g$, and $\tilde{x}$ to complete the proof. Note that no assumptions were made on the matrix $M$, hence showing that the result holds for arbitrary $M$ of appropriate dimension. Note further that the above holds true for any value of $\gamma$ for which (23) holds. In particular, the above proof holds for any value of $\gamma$ that is greater than the L2 gain of the full state system of Fig. 4 from which we conclude that the L2 gain of the full state system and the observer-based system are the same.

\section{ACKNOWLEDGMENT}

The authors would like to thank Dr. A. Megretski for his many useful comments on this work, along with Dr. P. Parillo for useful discussions on the numerical portion of this work, and the anonymous reviewers for the many comments they provided to increase the clarity of this exposition.

\section{REFERENCES}

[1] Z. Artstein, "Example of stabilization with hybrid feedback," Hybrid Systems III: Verification and Control, Lecture Notes in Computer Science, vol. 1066, pp. 173-185, 1996.

[2] C. Benassi and A. Gavioli, "Hybrid stabilization of planar linear systems with one-dimensional outputs," Syst. Control Lett., vol. 46, pp. 303-309, 2002.

[3] S. Boyd, L. El Ghaoui, E. Feron, and V. Balakrishnan, "Linear matrix inequalities in system and control theory," SIAM Studies Appl. Math., 1994.

[4] M. Dahleh, M. A. Dahleh, and G. C. Verghese, "Lectures on dynamic systems and control," Lecture Notes MIT Course, vol. 6.241, 1999.

[5] J. Gonçalves, "Constructive Global Analysis of Hybrid Systems," Ph.D. dissertation, MIT, Cambridge, MA, 2000.

[6] P. R. Gray and R. G. Meyer, Analysis and Design of Analog Integrated Circuits, 3rd ed. New York: Wiley, 1993.

[7] J. P. Hespanha, "Root-mean-square gains of switched linear systems," IEEE Trans. Automat. Control, vol. 48, no. 11, pp. 2040-2045, Nov. 2003.

[8] B. Hu, G. Zhai, and A. N. Michel, "Hybrid output feedback stabilization of two-dimensional linear control systems," in Proc. Amer. Control Conf., 2000, pp. 2184-2188.

[9] D. A. Johns and K. Martin, Analog Integrated Circuit Design. New York: Wiley, 1997.

[10] H. Khalil, Nonlinear Systems, 2nd ed. Englewood Cliffs, NJ: Prentice-Hall, Inc., 1996.

[11] D. Liberzon, "Stabilizing a linear system with finite-state hybrid output feedback," in Proc. 7th IEEE Mediterranean Conf. Control Automat., 1999, [CD ROM].

[12] E. Litsyn, Y. V. Nepomnyashchikh, and A. Posonov, "Classification of linear dynamical systems in the plane admitting a stabilizing hybrid feedback control," J. Dyn. Syst., vol. 6, no. 4, pp. 477-501, 2000.

[13] E. Litsyn, Y. V. Nepomnyashchikh, and A. Posonov, "Stabilization of linear differential systems via hybrid feedback controls," SIAM J. Control Optim., vol. 38, no. 5, pp. 1468-1480, 2000.

[14] A. Megretski, Lecture Notes for MIT Course 6.245. Cambridge, MA: MIT Press, 2002.

[15] A. Rantzer and A. Megretski, "A tutorial on integral quadratic constraints: Part I: IQC models and their feasibility," Personal Correspondence, 2002.

[16] K. R. Santarelli, A. Megretski, and M. A. Dahleh, "Stabilizability of two-dimensional linear systems via switched output feedback," Syst. Control Lett., vol. 57, no. 3, pp. 228-235, 2008.

[17] K. R. Santarelli, M. A. Dahleh, and A. Megretski, "Optimal controller synthesis for second order LTI plants with switched output feedback," in Proc. 46th Conf. Decision Control, 2007, pp. 4127-4132.

[18] K. R. Santarelli, "On the Synthesis of Switched Output Feedback Controllers for Linear, Time-invariant Systems," Ph.D. dissertation, MIT, Cambridge, MA, 2007.

[19] X. Xu and P. J. Antsaklis, "Design of stabilizing control laws for second-order switched systems," in Proc. 14th IFAC World Congress, 1999, vol. C, pp. 181-186.
[20] X. Xu and P. J. Antsaklis, Stabilization of Second-Order LTI Switched Systems Dept. Elect. Eng., University of Notre Dame, ISIS Tech. Rep. isis-99-001, 1999.

[21] G. Zhai, B. Hu, K. Yasuda, and A. N. Michel, "Disturbance attenuation properties of time-controlled switched systems," J. Franklin Inst., vol. 358, no. 7, pp. 765-779, 2001.

[22] G. Zhai, H. Lin, and Y. Kim, "L2 gain analysis for switched systems with continuous-time and discrete-time subsystems," in Proc. SICE'04 Аnпи. Conf., 2004, pp. 658-663.

[23] J. Zhao and D. J. Hill, "On stability and L2 gain for switched systems," in Proc. 44th Conf. Decision Control, 2005, pp. 3279-3284.

[24] K. Zhou and J. Doyle, Essentials of Robust Control. Englewood Cliffs, NJ: Prentice Hall, 1998.

[25] K. R. Santarelli and M.A. Dahleh, "Comparison between a switching controller and two LTI controllers for a class of LTI plants," Int. J. Robust Nonlin. Control, vol. 19, pp. 185-217, 2009.

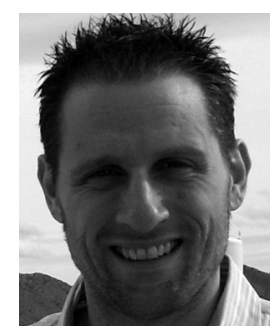

Keith R. Santarelli (M'07) was born in 1977. He received the S.B., M.Eng., and $\mathrm{Ph}$. D. degrees in electrical engineering and computer science from the Massachusetts Institute of Technology, Cambridge, in 1999, 2000, and 2007, respectively.

Since then, he has been with the Electrical and Microsystems Modeling Department, Sandia National Laboratories, Albuquerque, NM, where he is currently a Senior Member of the Technical Staff. His research interests lie in the area of systems and control, including the design of switched and hybrid control architectures, optimal control theory, model order reduction, and associated computational techniques. In particular, he is interested in problems which lie on the boundary of systems theory and which can be applied to the areas of circuit design, simulation, uncertainty quantification, and signal processing. He is also interested in the area of synthetic biology, and, in particular, the application of systems theory to the design of interconnected biological systems for engineering purposes.

Dr. Santarelli received the Harold L. Hazen Award for Teaching Excellence in 2003, the MIT EECS Special Recognition Award in 2007, and the R\&D 100 Award for the Xyce Parallel Electronic Circuit Simulator in 2008.

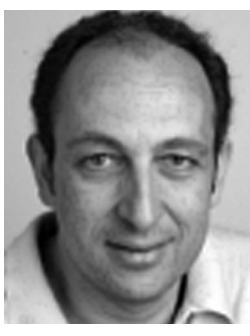

Munther A. Dahleh (S'84-M'87-SM'97-F'01) was born in 1962. He received the B.S. degree from Texas A \& M University, College Station, in 1983 and the Ph.D. degree from Rice University, Houston, TX, in 1987, all in electrical engineering.

Since then, he has been with the Department of Electrical Engineering and Computer Science, MIT, Cambridge, MA, where he is now a Full Professor. $\mathrm{He}$ has been a Visiting Professor at the Department of Electrical Engineering, California Institute of Technology, Pasadena, for the Spring of 1993. He has held consulting positions with several companies in the U.S. and abroad. $\mathrm{He}$ is the co-author of Control of Uncertain Systems: A Linear Programming Approach (Englewood Cliffs, NJ: Prentice-Hall) and the co-author of Computational Methods for Controller Design (New York: Springer). He is interested in problems at the interface of robust control, filtering, information theory, and computation which include control problems with communication constraints and distributed mobile agents with local decision capabilities. He is also interested in model reduction problems for discrete-alpahbet hidden markov models and universal learning approaches for systems with both continuous and discrete alphabets. He is also interested in the interface between systems theory and neurobiology, and in particular, in providing an anatomically consistent model of the motor control system.

Dr. Dahleh received the Ralph Budd Award in 1987 for the best thesis at Rice University, the George Axelby Outstanding Paper Award in 1987, an NSF Presidential Young Investigator Award (1991), the Finmeccanica Career Development Chair (1992) and the Donald P. Eckman Award from the American Control Council in 1993, the Graduate Students Council Teaching Award in 1995, the George Axelby Outstanding Paper Award in 2004, and the Hugo Schuck Award for Theory. He was a plenary speaker at the 1994 American Control Conference, at the Mediterranean Conference on Control and Automation in 2003, and at the MTNS in 2006. He was an Associate Editor for the IEEE TRANSACTIONS ON AutOMATIC CONTROL and for Systems and Control Letters. 